\title{
A Reuseable Framework for Designing Socially Assistive Robot Interactions
}

\author{
Sebastian Schneider, Michael Goerlich and Franz Kummert \\ Applied Informatics, CITEC, Inspiration 1, 33602 Bielefeld
}

\begin{abstract}
Robots are increasingly tested in different socially assistive scenarios. Future applications range from dieting, coaching, tutoring to autism therapy. In such applications the success of the system is commonly evaluated by the ability to encourage the user to keep up with a task. Hence, one important requirement for supportive systems is to have an interactional motivational model that formalizes the way how users can be assisted. In this paper we describe our framework for coordinating motivational interaction scenarios with socially assistive robots (SAR) in the context of sport assistance. We exemplify three different sport scenarios where we have used the same motivational interaction model. Furthermore, we show how this model can be used to systematically test the different aspects of motivation in the context of SAR in sport domains. Therefore, we have conducted an experiment to evaluate the importance of acknowledgement from SAR for human interaction partners. The results show that users exercise longer if acknowledgment is included into the motivational model.

Keywords: Human-Robot Interaction, System Design, Socially Assistive Robots
\end{abstract}

\section{Introduction}

Research in Socially Assistive Robotics $(S A R)$ targets scenarios where robots instruct people during tasks that benefit from some social assistance like rehabilitation, dieting or cognitive tasks [8, 13, 15]. Those systems are often built 
5 from scratch and implemented interaction patterns are hand-crafted for each scenario or new application. This leads to recurring implementations of interaction structures that are difficult to compare across different systems or use cases. If we take a closer look, we see commonalities between the different scenarios that require social assistance. In the examples mentioned above we see that in all scenarios users are working towards a task goal. While the tasks (e.g. dieting, rehabilitation, cognitive or engagement tasks) are different, all share common attributes (see Table 1). They have a beginning, measurable parameters and a goal. Thus, the social assistance relates to these values and the task goals and support (e.g. acknowledgment, feedback or reparation) can be triggered to help the user reach the goal. The question is if there are common motivational patterns that capture the interactional requirements neccessary to keep a user motivated to work on a task. And if so, can this concept help to systematically test the important aspects of social support?

In previous work we have developed a motivational interaction model which we have evaluated in an extended long-term study [25]. While the previous implementation focused on a single use case, we have now worked on the reusability of this model in the scope of a modular framework. In this work we describe a general formalized framework for SAR and introduce three scenarios that made use of it. Furthermore, we want to show that the modularization of the motivational interaction patterns allows to systematically test different aspects of interactional motivation.

We propose that re-usability of common motivational concepts and frameworks could help to systematically carry out experiments, measure the scientific progress and be reused in other domains.

30

The paper is organized as follows: First we will give a brief introduction of motivation as a key component for building SAR robots. Afterwards, we will explain our prior research efforts in this domain. In Section 3 we explain our current framework for designing SAR robotic scenarios. In Section 4 we will show the current usage of our framework as well as an evaluation on how this framework can be used to verify the importance of different interactional aspects 
of our modular motivational interaction patterns. In the last section, we will give a conclusion.

\section{Related and Previous Work}

\subsection{Motivation: A Key Component for SAR}

To develop a common concept of motivational support for SARs it is indispensable to identify the key components of motivation from an interdisciplinary perspective. There exists a wide variety of different motivational theories. For example, J. E. Barbuto [10] discriminates motivation in extrinsic and intrinsic motivation with further subdivisions. Motivation can also be influenced by the

45 goals for a task [16] and the intention to show a certain behavior can be influenced by the expectations of a a significant other [1. Lastly, motivation is varied by a person's high or low self-efficacy belief toward the behavior [2]. Besides the amount of different motivational theories that could be applied to SAR recent research has mostly incorporated intrinsic motivation (and specially the theory of flow [6] ) for their task assistance to adapt the task difficulty to match the user's individual optimal challenge [7, 17. The general definition claims that motivation is a force which drives human behavior but this perspective focuses mostly on the internal states of an individual person. However, in socially assisted scenarios one main goal is to collaboratively achieve a goal. Therefore, also a sociological and linguistic perspective which analyze the different multimodal cues during interactional processes have to be considered. Some form of communication which helps express one's desires and intentions has to be established. Therefore, future systems need to deal with miscommunication, need to have repair mechanisms and require a concept of when to trigger which kind of supportive feedback in a multi-modal manner in order to achieve a goal-oriented interaction [21].

In conclusion, the diversity and complexity of the different motivational theories show that it is a challenging task to apply one that could help to keep a trainee motivated to exercise. Depending on the task, the user group or the 
environment a different kind of theory might be suitable. Thus, it is difficult to implement a single theory of motivation into a SAR system because both a global and a local view on motivation are important. However, to narrow the current work we are focusing only on local motivation between a trainer and a trainee from an intstructional perspective.

\subsection{Approaches in SAR Systems}

How did other researchers tackle the problem of incorporating motivation in their work on SAR? Jayawardena et al. [11] propose a three layered architecture for rapid prototyping of SAR systems and easy to use behavior description for subject matter experts (SME). A similar approach was reported from Mead et al. 18. However, both approaches focus on the realization of an architecture and not on a formalized behavior description for motivational instruction patterns robots could use to provide support. In these cases motivational instructions are designed by some experts. Others focus on reinforcement learning approaches to learn which behavior is motivating the user the most by e.g. reducing the user's so stress or changing the user's valence [5, 14]. Leyzberg et al. [15] proposes the usage of bayesian models to provide a suitable assistance based on the user's task experience. Looking at these examples one could wonder whether there is no connection between the different scenarios and believe that there exists no common pattern that could model the motivational interaction. In Table

${ }_{85} 1$ we have summarized the different tasks, measures and supportive behaviors of a selected number of publications. The most prominent supportive behavior those systems provide is offering suggestion (e.g. advises, corrections, help, repair). The second most offered support is giving encouragement and praise (e.g. positive feedback, acknowledgment). Hence, these systems observe the 90 task parameters and trigger supportive behavior for the users. If the users fail on a task they provide encouragement and in case they succeed acknowledgment. However, in all of the publications it is not described how the transition from one behavior to the other is modeled and whether there is a formalized connection between the different assistive behaviors. 
Table 1: Comparison of different tasks, measures and supportive behavior in SAR scenarios.

\begin{tabular}{|c|c|c|c|}
\hline Reference & Task & Measures & Supportive Behavior \\
\hline Kidd and & dieting & daily calory income and ex- & suggestions, advices \\
\hline Breazeal [13] & task & ercising & \\
\hline $\begin{array}{l}\text { Leyzberg et al. } \\
15\end{array}$ & $\begin{array}{l}\text { nono- } \\
\text { gram }\end{array}$ & $\begin{array}{l}\text { puzzle state, time, skill as- } \\
\text { sessment score }\end{array}$ & $\begin{array}{l}\text { instructions, } \quad \text { strategy } \\
\text { lessons }\end{array}$ \\
\hline & puzzle & & \\
\hline $\begin{array}{l}\text { Chan and Nejat } \\
\text { [5] }\end{array}$ & pairs & puzzle state, stress & $\begin{array}{l}\text { instruction, help, acknowl- } \\
\text { edgment, encouragement }\end{array}$ \\
\hline Leite et al. [14] & chess & $\begin{array}{l}\text { winning, game state, get- } \\
\text { ting better/worse, valence }\end{array}$ & $\begin{array}{l}\text { encouragement, feedback, } \\
\text { suggestions }\end{array}$ \\
\hline Midden and & laundry & energy consumption & positive/negative social \\
\hline Ham 19 & & & feedback \\
\hline Fasola and & exercise & arm position & corrections, praise, guid- \\
\hline Matarić [7] & games & & ance, encouragement \\
\hline $\begin{array}{l}\text { Schneider et al. } \\
{[22}\end{array}$ & $\begin{array}{l}\text { mental } \\
\text { rotations }\end{array}$ & correct answers, time & guidance, suggestions \\
\hline
\end{tabular}

In this work we want to expand the field by introducing a framework and motivational interaction patterns which can be used and reused to systematically study the motivational concepts that SAR require. However, at the current stage of our work we can not consider the whole range of different domains in which SARs can be used. Therefore, we restrict our work to the domain of sport assistance.

\subsection{Previous Work}

We have investigated the instructional structures and motivational strategies that trainers incorporate into everyday workout (i.e. indoor cycling) in real world Human-Human Interaction. During field investigations colleagues observed the interaction between a coach and an athlete during indoor cycling sessions. The goal of the investigation was to identify some common interactional patterns that coaches use to motivate and engage their athletes 24]. A 


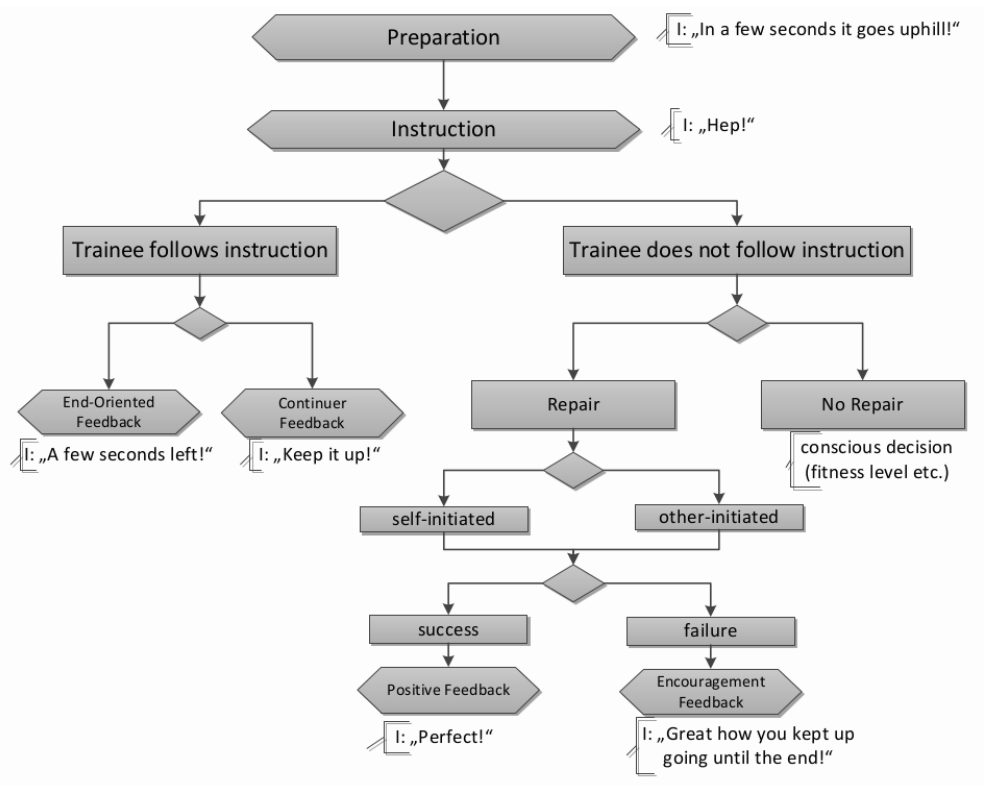

Figure 1: Interactive action-based motivation model [25].

qualitative analysis revealed a complex multi-modal structure of motivationrelevant processes that are fine-grained and sequentially. This model was reduced to an interactive action-based motivation model due to the limitations of current robotic systems (see Fig. 1). It captures the aspects of preparation, instruction, acknowledgment, repair and feedback (i.e. continuer-, encouraging-, positive-, end-oriented-feedback) in a systematic way for single exercise instructions/movements.

Concerning the five design principles for SAR from Fasola and Matarić 7 ] (i.e. motivation, fluid interaction, personalization, intelligent behavior and task driven behavior) this model captures some of them from a conceptual point of view. The model as a whole satisfies the requirements of fluid interaction by structuring exercises and guiding the user through the tasks in a formalized manner. The feedback fulfills the requirement for motivation by giving positive feedback if the user reaches the goal of an exercise or guidance and encouragement if the user does not reach the intended goal of an exercise. The reparation 
is adapted towards the user's activity and personalized by the user's fitness level. At last, the exercises need to be defined in a specified format including the exercise goals to match the requirement for a task driven behavior. This is fulfilled by the preparation which can incorporate the exact exercise goals.

Since our previous implementation was tailored for a single use case we will describe how we model the conceptual requirements in a framework that suits a variety of sport scenarios.

\section{A Formalized Framework for Socially Assistive Robots}

Our framework (see Fig. 2p consists of four main components that communicate over the Robotic Service Bus (RSB) middle-ware [26]. The communication between the components is based on a publish-receive pattern and on remoteprocedure calls. The messages can include different RSB Data Types (RST ${ }^{1}$ ). The framework is composed of a state-machine based scenario description, a decision server that triggers state changes based on the sensor input, data pipelines receiving sensor information, and dialog acts. We illustrate our framework by going through the different parts using a simple example scenario (i.e. a user is asked to raise an arm and hold it for some seconds).

\subsection{Motivational Instruction Patterns}

As outlined in Section 2.3, we have identified recurring patterns that describe motivational instructions. They represent sequences of states that characterize a socially assistive task and provide a reusable solution for scenario implementations. It is a graphical model that captures the interaction between a trainee and a trainer as well as the system level. Thus, it serves as a guideline for developers and as internal interaction model.

We distinguish two types of interaction patterns: A static movement pattern which represents tasks requiring to do some static exercises (e.g. cycling with predefined speed, see Fig. 3. And a cyclic movement pattern which represents

${ }^{1}$ http://docs.cor-lab.de//rst-manual/trunk/html/data-types.htmll 


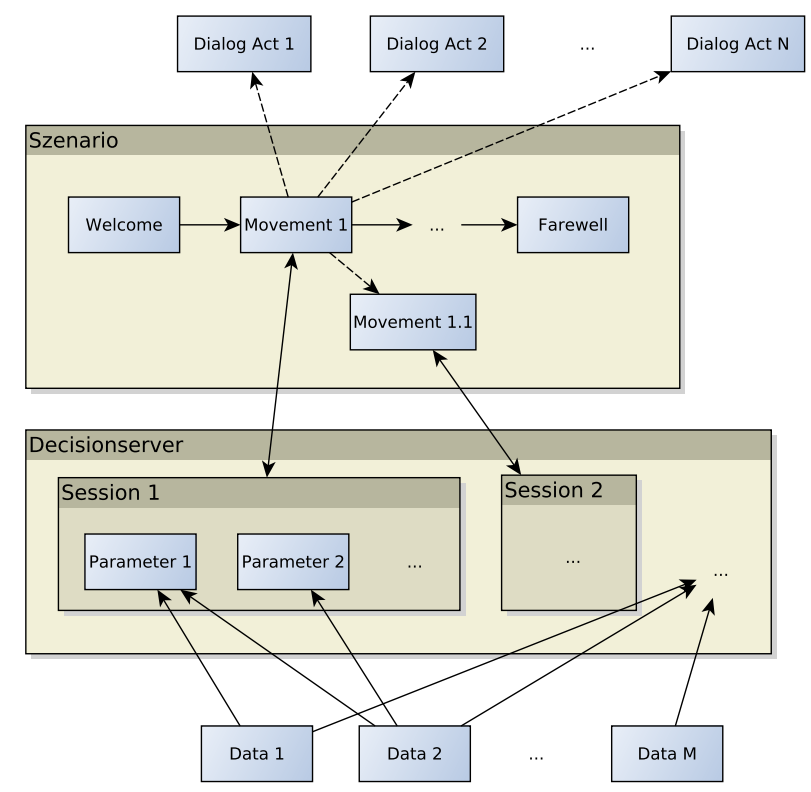

Figure 2: Framework for a socially assistive scenario implementation.

tasks where the user has to follow a cyclic path (e.g. doing squats, see Fig. 4). Considering our example scenario raising an arm and holding it for some time is a static movement. Pattern instances require a variable context with dynamic as well as static information that are provided by a configuration in XML format. This includes the utterances for each state, parameters for the decision component specifying exercise targets, configurations for the evaluation and ending of movement. Table 2 summarizes the different states of the patterns. We incorporated a high flexibility and modularization in our implementation so that a variety of scenarios can be realized. For example, teaching and coaching scenarios require to trigger exercises that can correct a wrong exercise execution (i.e. the system can help the user to reach a specific pose required for an exercise or to start a correcting exercise). Thus, it is important that each state can also activate other state machines or instruction patterns in a hierarchical way:

1. Hierarchy States can trigger simple utterances, dialog acts and also new movement pattern (see Fig. 2, Movement 1 initiates Movement 1.1). 


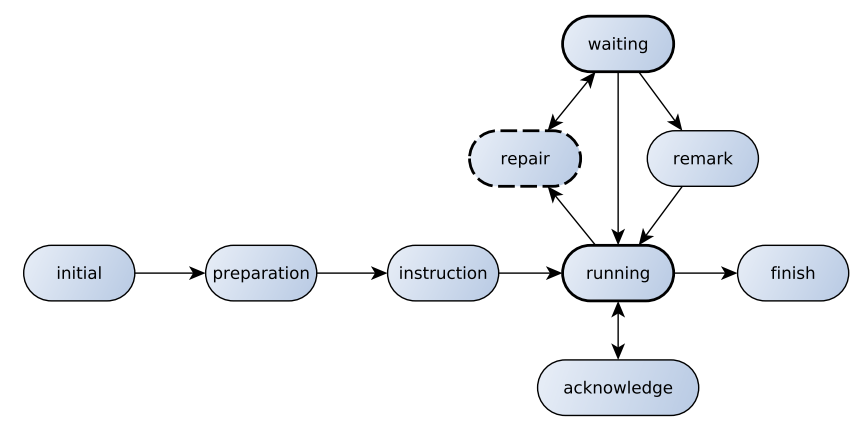

Figure 3: Static Movement Instructions.

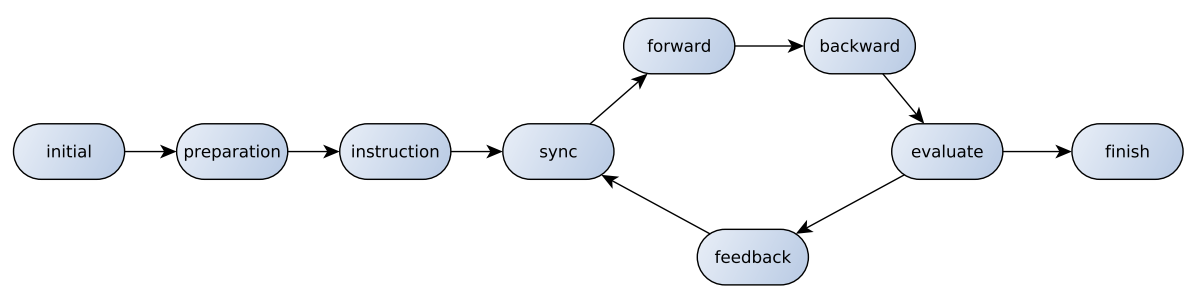

Figure 4: Cyclic Movement Instructions.

In some scenarios it might be necessary to synchronize the actions between the system and the user.

2. Synchronization Cyclic movements include a synchronization point and a waiting task.

During the wait task the decider verifies if the user has reached the desired position. If the user does not comply, the system can continue with the execution of the next cycle or it can start a reparation. For certain exercises the order of different states is important and one synchronization point is not sufficient.

3. Ordering Cyclic movements can have forward and backward actions with multiple synchronization points (see Figure 5).

As seen in the related work, it is not necessary to always include every state of the pattern. In some cases acknowledgment is sufficient in other cases only reparations might be useful.

4. Modularization States are modular and can be omitted. 
Table 2: Overview and explanation of the different states.

\begin{tabular}{|c|c|}
\hline static pattern & explanation (example utterance) \\
\hline preparation & $\begin{array}{l}\text { Preparation for the next exercise (Next we will do an arm raising } \\
\text { exercise. Please sit down.). }\end{array}$ \\
\hline instruction & $\begin{array}{l}\text { Instructions for the next exercise (Now copy my movement for } 20 \\
\text { seconds.). }\end{array}$ \\
\hline running & Observation of the exercise targets \\
\hline repair & User does not reach the target (Raise the arm a little bit higher). \\
\hline waiting & Evaluation of the reparation (Still a little bit higher). \\
\hline remark & The user is not able to reach the target (Good try!). \\
\hline acknowledge & User reaches the target (You are doing it right.). \\
\hline finish & $\begin{array}{l}\text { Finishes the exercise after a specified termination parameter (We are } \\
\text { done. Nice work.). }\end{array}$ \\
\hline cyclic pattern & explanation \\
\hline sync & $\begin{array}{l}\text { Synchronizes the action of the user and the system (e.g. the system } \\
\text { waits for the user to finish a cycle) }\end{array}$ \\
\hline $\begin{array}{l}\text { forward- } \\
\text { backward }\end{array}$ & Ensures a correct ordering of the cyclic path. 5 \\
\hline evaluate & After a cycle the performance of the user is evaluated. \\
\hline feedback & Based on the evaluation the system can give a feedback. \\
\hline
\end{tabular}

180 Technically, the movement patterns are modeled in a Domain Specific Language (DSL), which is translated into statecharts [23] in the SCXML format and executed by a scenario coordination that uses the Apache Commons SCXML engine ${ }^{2}$,

\subsection{Dynamic Decision Component}

On movement initialization the scenario coordination starts a new session on the decision server. This server manages three aspects: A data-processing

\footnotetext{
2 https://commons.apache.org/proper/commons-scxml/
} 


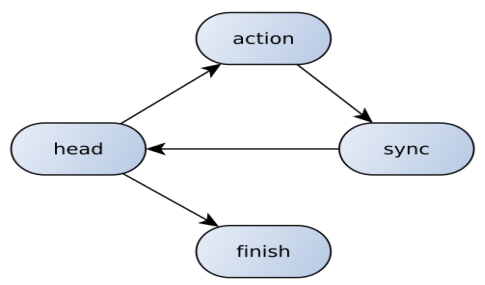

(a) The act-action.

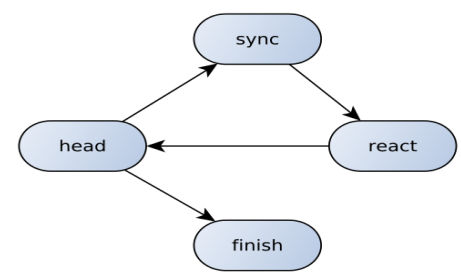

(b) The react-action.

Figure 5: Two possible actions for the forward-backward states of the cyclic movement. In act-actions the robot starts the exercises and waits for the user to follow. During reactactions, the robot follows the user's lead.

pipeline, local-global decisions and evaluation-finishing strategies. Based on the configuration the server makes decisions during movement run time (see Figure 2). Those decisions trigger a transition between the different states in the movement pattern.

As seen in Table 1 the assistance is either based on data representing a task state or a measurement of the user (e.g. strings, numbers, classification results). Because the values to decide on are inherently different between scenarios we have implemented a data-processing pipeline that defines input- and output data slots.

\subsubsection{Configurable Data-Processing Pipeline}

The pipeline consists of three main blocks: data sources, transformations and deciders. Data sources are input-slots that receive specific data types on a required format (e.g. skeleton data to vector objects and joint angles) or calculate descriptive statistics on the incoming values (e.g. running median or means). At last, deciders transform in-slots to decision results or filter decisions of other components (i.e. in-range decider, entropy decider). into a joint angle format on which a descriptive statistic can be calculated. A 
decider evaluates whether the incoming data is in a specified range and, in the case we don't care which arm is raised, the last decider can evaluate whether one of the deciders provided a positive evaluation.

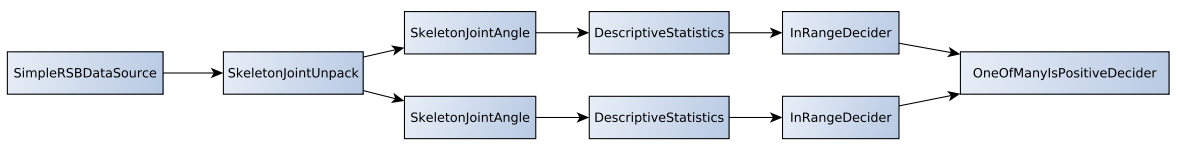

Figure 6: Processing pipeline for the use case scenario.

\subsubsection{Local and Global Decisions}

Local decisions are represented as a decision reason including the name of the parameter, the local decision, a time stamp and a boolean variable showing whether a goal is reached. In our use case this could be the name of the parameter (e.g. "shoulder angle), the local decision could be "too low" if the value is below a specified threshold ("too high" or "equal" respectively) and false (or true) for the goal violation. They are collected into a decision bag (see Fig. 7) which is verified by a global decider. This decider can trigger a specific supportive behavior based on the collected decisions. Current implemented deciders are a simple decider, a hierarchic reaching decider and a hierarchic monitoring decider. The simple decider evaluates the decision bag for errors. If an error is found, a repair advice is sent and the guidance is set to failed. If there is no error, an acknowledge is sent. Decisions on multiple concurrent parameters can be handled by the hierarchic reaching decider. Lastly, the hierarchic monitoring decider is similar to the hierarchical reaching decider, but it observes the specified parameters for a longer range of time.

\subsubsection{Evaluation and Finishing Strategies}

Finally, we have implemented strategies to finish a movement or to start the evaluation of the decision bag. They are separated into distinguished strategies to meet the different requirements of varying scenarios (see Fig. 7). The movement can be evaluated or finished manually, after a certain amount of time, 


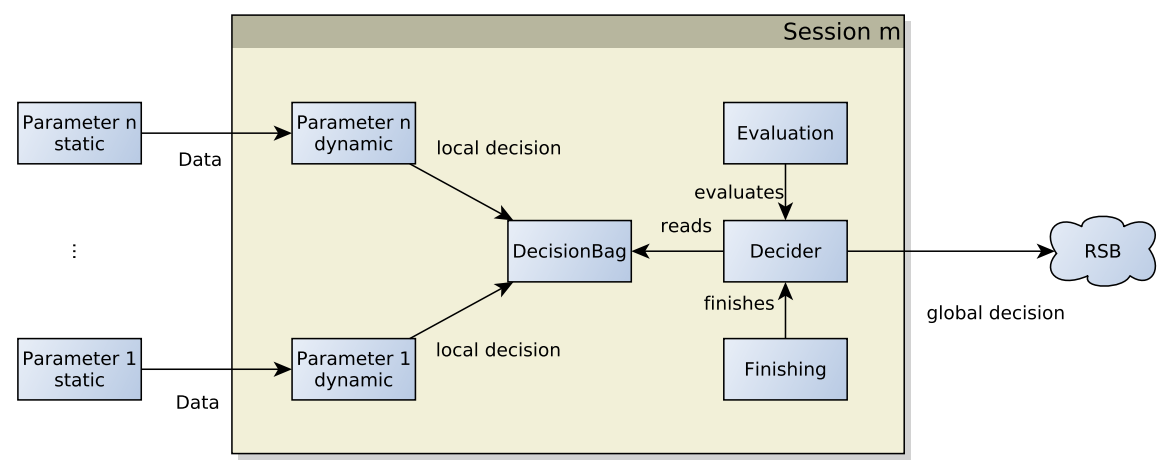

Figure 7: Overview of the decision system.

after a number of events or by a external component. Regarding the use case scenario, the evaluation strategy could be set to 10 seconds and the finishing strategy to 20 seconds. Thus, if the user is able to reach the required arm position and can hold it $\mathrm{s} /$ he will receive an acknowledgment after ten seconds and the movement finishes after twenty seconds have passed.

The evaluation strategy can give five different types of guidance: continue, no reason for a change in the current situation; repair, reasons make a reparation necessary; acknowledge, reasons favor a praise; finished, last known state was accurate and failed, last known state required a reparation.

\subsection{Scenario Coordination}

The scenario coordination is a state machine in which the whole interaction flow can be modeled. This includes generic robot behaviors (idle behavior, greeting, farewell, robot navigation) and task related movement patterns. To help non-expert users to create a SAR scenario state machine and robot programming are implemented in a Domain Specific Language (DSL) which is automatically transformed to SCXML. It eases the process of handling the middleware communication between the different parts of the system and therefore simplifies the programming effort (for details regarding the middle ware see Section V of 20]). Additionally, DSLs have the benefit of providing specified input format and suggestions on how to program a scenario for the user. Moreover, it reduces 
errors by including auto-completions and constraints. As implementation tool we use the Meta Programming System developed by JetBrain $\$^{3}$

While the scenario and movement patterns are modeled in a DSL the configuration of the movements are in XML format. The configuration includes the dialog acts that are triggered in the different states of a movement, the exercise targets (e.g. joint angle configuration of the user, speed or number of repetitions of exercises) and which deciders and strategies should be used.

\subsection{Movement Pattern as Building Blocks for Socially Assistive Interactions}

260

How can this framework be used to create a work flow that helps to build new scenarios? Designers need to ask themselves the following questions: Does the user perform a static task or a cyclic task? If it is a cyclic task, how many steps does a cycle have? What measures exists to detect a correct transitioning through the different states? What values need to be observed? Are reparations or acknowledgments necessary? If yes, what kind of reparation should be given based on input? When does an exercise finish? After a specified amount of time, after a number of correct cycles or after the user stops?

If these aspects are considered, the interaction design is only a concern of right configuration and implementation of a suited detection or recognition system. In the following we describe our attempts to create different assistive scenarios with the same framework and describe where we needed to implement some extensions.

\section{Usage and Model Evaluation}

Indoor Cycling. In this scenario (see Fig. 8a the robot is instructing the user 275 to cycle at different speed, resistance and in different positions (e.g. standing, sitting or doing push ups on the bike). Each movement is finished after a specific time which is based on the length of a song that is played during the indoor cycling session. An earlier version of our framework and the motivational

$\sqrt[3]{\text { https : //www.jetbrains.com/mps / }}$ 


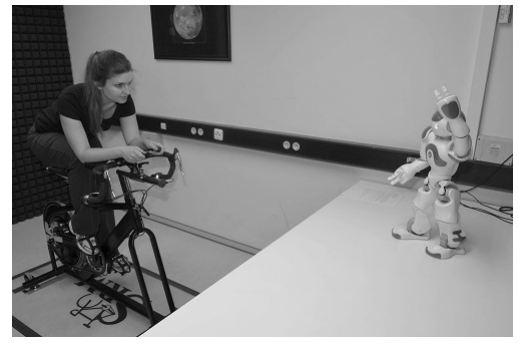

(a) Nao as spinning instructor.

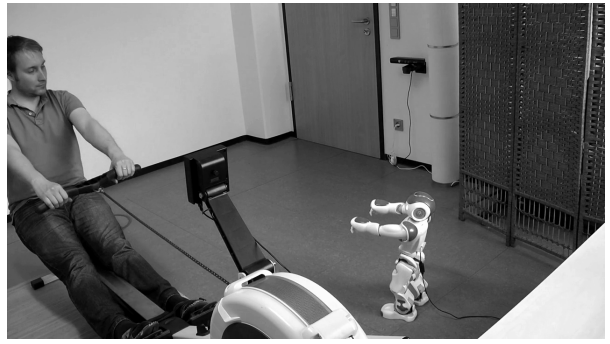

(b) Nao as rowing instructor.

Figure 8: Example scenarios.

instruction model have been evaluated during a 18-days long-term study 25. In hierarchy. We reused the data-processing pipeline, the scenario coordination and the decision server in this scenario. However, we needed to implement new activity recognition systems to evaluate the position of the back and legs of the 


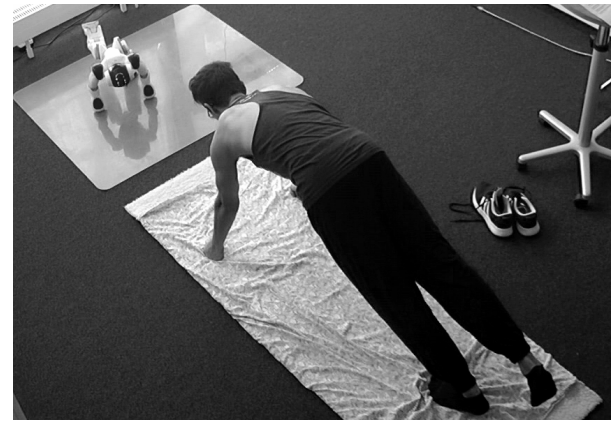

(a) Nao as bodyweight workout instructor.

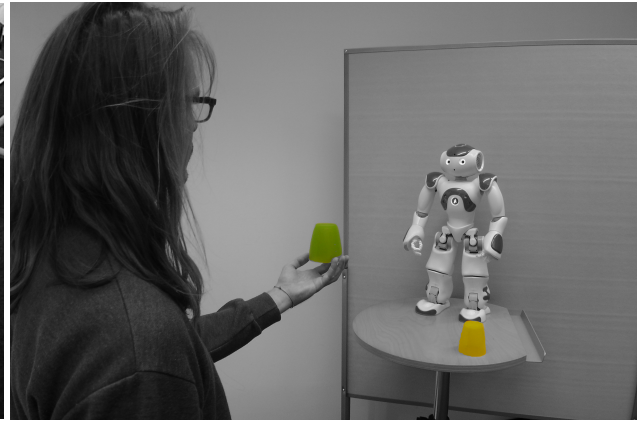

(b) Toy scenario: Nao teaching colours.

Figure 9: Example scenarios.

backed to the user. For the cases where the user is leading the exercising we implemented a new dialog act that allows the robot to ask the user whether $\mathrm{s} /$ he wants to quit.

For all scenarios we used the same robot (i.e. Nao) in order to exclude effects 

possibility of creating a variety of different scenarios. Furthermore, it could be easily extended to create a full interactive system that could help e.g. children to learn colours.

Still, a detailed analysis of the applicability is needed and in the future we

\subsection{Towards a Systematic Model Evaluation}

While we can not give a quantitative evaluation on the applicability of our framework, we can show how this model can be used to systematically test different motivational aspects. 


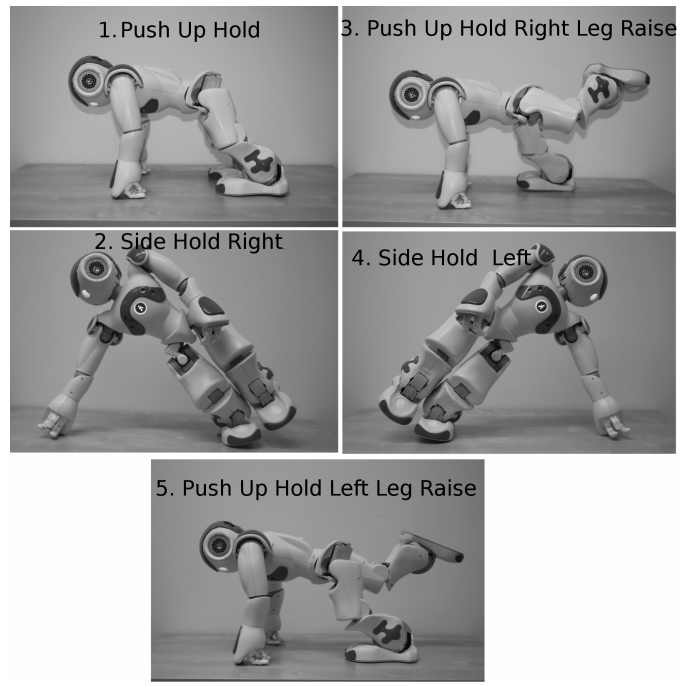

Figure 10: Five isometric exercises from the body weight training.

The modularization of the different states of the interactional model allow to systematically test the importance of different social support states (e.g. repair, acknowledgment). To evaluate the different parts of the instructional model we have implemented a SAR system that instructs users to do five isometric exercises (see Fig 10). To evaluate the importance of the acknowledgment state on people's motivation to hold these exercises we have conducted a study where we compare the static movement pattern including only the acknowledgment and one where we also excluded acknowledgment (see Fig. 11).

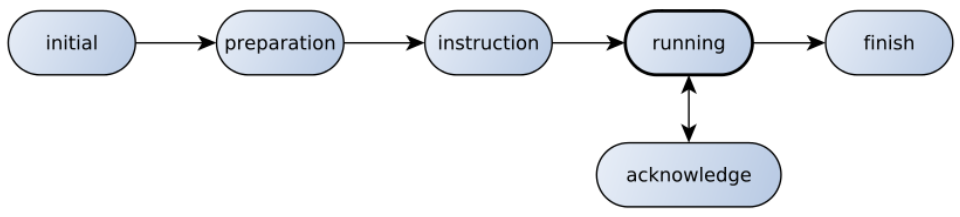

(a) Static movement including only acknowledgment.

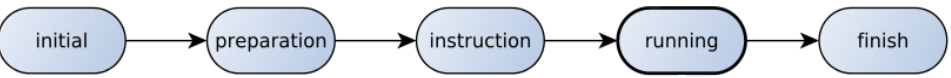

(b) Static movement with no repair and no acknowledgment.

Figure 11: The two static movement configurations for the study design. 


\subsubsection{Study Design}

Participants had to do two blocks of five isometric exercises each (see Fig.

360 can not persist the exercises anymore they were told to stand up, wait for thirty seconds and then start the next exercise. In each condition the participants did not know that they have to do a second exercise block. In the first block the participants did the exercises alone and in the second block either alone or with was announcing the exercises the user had to do, as well as how long the break is. In the robot instructor feedback (RIF) condition the robot was additionally giving an acknowledgment to the user based on their performance from the first block. After three quarters of the time they held the exercises during the first holding the exercises. As measurements of the importance of acknowledgment for the motivation to exercise we have used the Godspeed questionnaire [3] as well as the duration how long they persisted the exercises compared between the first and the second block. This study design was inspired and adopted by $375 \quad 9$.

\subsubsection{Experimental Design and Participants}

Participants $(\mathrm{n}=50)$ were assigned to one of three conditions (17 in independent condition (IC), 17 in robot instructor condition (RI) and 16 in robot instructor feedback condition (RIF)). Participants were mostly students (male: 32, female: 24 , age $\mathrm{M}=26.05$ years, $\mathrm{SD}=6.12$ ) from our university. They received seven Euros as monetary compensation.

\subsubsection{Procedures}

The participants arrived at the lab individually, read and signed a consent form which informs them that they will be recorded during the experiment.

\footnotetext{
${ }^{4}$ https://www.aldebaran.com/en
} 

guided to the lab and told to start after they have waited for a short time, so that the experimenter can check that the recording is working properly. Then the participants did each exercise alone in the lab while the experimenter observed them from a different room and took the times of each exercise. The participants completed Block 1 (each exercise once). Afterwards, the participants had a ten minute break where they were offered a glass of water. After the break participants in the IC condition were told the average time they held the planks and that they would complete the same set of exercises again (Block 2). In every condition the participants were not told that they had to do a second block of exercises until they had finished the first block. During the robot conditions participants were told that they will do the same set of exercises again but that this time a robot will be present. They were instructed to follow the guidance of the robot through the session. Participants were told the average time they held the planks, but received a false information on how long the robot can due to prior research which showed that people treat agents more like humans when there was an initial verbal interaction between them [4]. After Block 2 the robot thanked the participants for their participation, told them that they are allowed to leave the room, that it needs to rest a bit and powered itself down. After leaving the room the participants completed a questionnaire, were debriefed and received a monetary compensation. The whole procedure took about 45 minutes to one hour. 


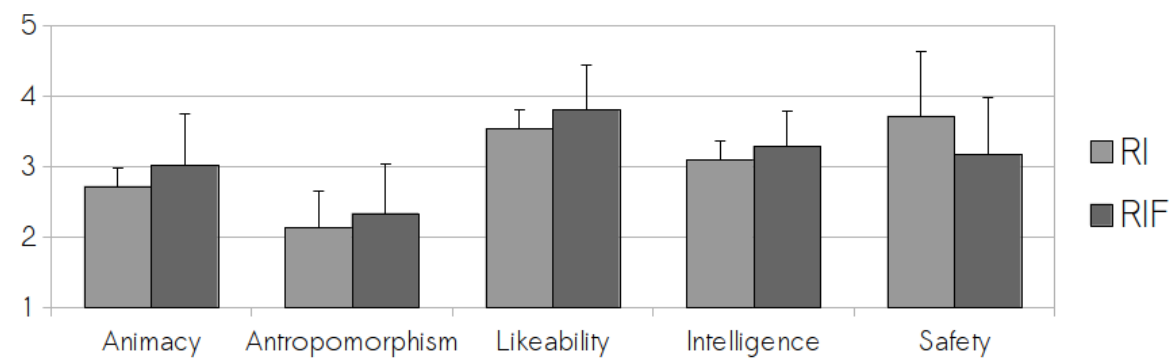

Figure 12: Godspeed scales and training enjoyment.

\subsubsection{Measures}

Persistence. Persistence was the number of seconds a plank was held from the moment participants moved into position until they quit. Block scores were calculated using total average seconds held on all five exercises.

Godspeed Questionnaire. In order to asses different perception of the robot between the conditions we asked the participant to rate the robot based on the Godspeed questionnaire (5 point-based differential scale, [3]).

Physical Training Enjoyment. We assessed the physical training enjoyment the users had using the Physical Activity Enjoyment Scale ([12]). We used the average value of all items as overall enjoyment score. Furthermore, we asked them about their intention to train tomorrow for at least 30 minutes.

\subsubsection{Results}

The results of the Godspeed questionnaire are depicted in Figure 12 , A Multivariate Analysis of Variance (MANOVA) revealed no difference between the conditions on the Godspeed Questionnaire $\left(F_{1,32}=0.45, p=.50\right)$.

As a primary dependent variable we used the average difference persistence time in seconds between the two blocks (Block 2 - Block 1). This approach controls for individual differences in strength and fitness and shows possible changes in persistence. The results obtained for the average block score of Block 2 subtracted with the average block score of Block 1 are shown in Figure 


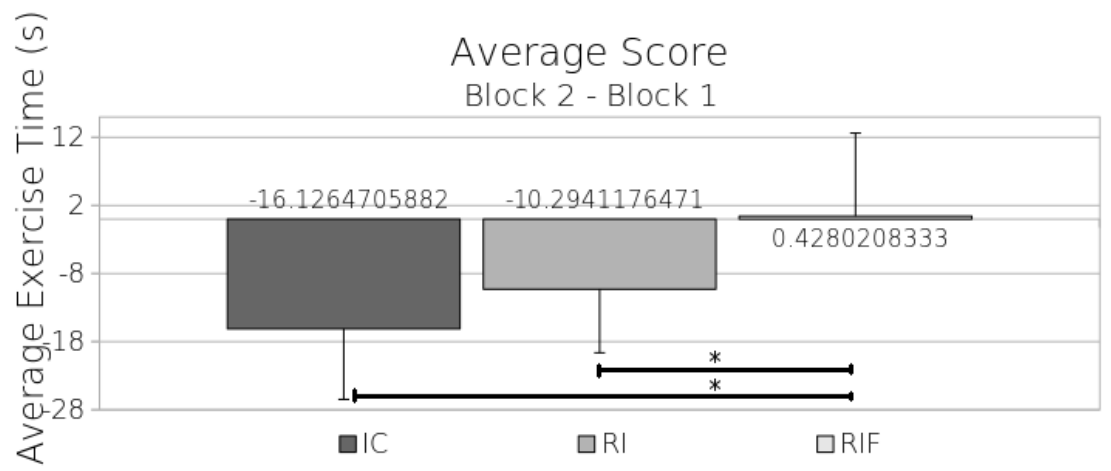

Figure 13: Block scores

13. An Analysis of Variance (AOV) on the difference score showed a significant main effect for the conditions $(F(2,51)=11.33, p<0.001)$. A Tukey's

$S D=12.2)$ and IC $(M=-16.12, S D=10.35)(p<0.001)$, and between RIF and RI $(M=-10.3, S D=9.34)(p<0.05)$. Additionally, we found no differences in the average Block 1 scores $(\mathrm{F}(2,51)=1.13, \mathrm{n} . \mathrm{s})$. Thus the results are not due to any general higher fitness level in the RIF population.

An AOV also showed no differences in training enjoyment $(\mathrm{F}(2,51)=1.837$, n.s.) and sport per week $(\mathrm{F}(2,51)=0.13$,n.s.). However, we found a difference for the intention to exercise $(F(2,51)=1.93, p<0.05)$. A Tukey's pairwise comparison revealed a significant difference between the RI and IC condition $(p<0.05)$.

\subsubsection{Discussion}

First of all, the results show that the framework allows to systematically test the importance of different motivational aspects for SAR. The implementation of both interactive behaviors was fairly easy and only included to dynamically change the acknowledgment time of each exercise suited to each user. Therefore, we were able to verify a new important research question. The results show that acknowledgment is an essential part of the instructional model and should be included in every implementation of an SAR system. Even though the subjective ratings did not differ significantly, the objective task performance 
shows that the motivation to exercise is higher in cases where the system gave acknowledgment compared to a system which did not give acknowledgment and to the IC condition.

A significant difference in performance was measured but no differences for intention and enjoyment were found (at least between the RIF and all other conditions). Participants persisted longer without any effects on enjoyment and future exercise plans. These results show that it is possible to extend exercising time without negative effects.

To further investigate the importance of different social support states we will conduct a follow-up study testing whether users have an additional motivational boost when the system is repairs wrong exercise execution.

\section{Conclusion}

In this paper we have presented our proposed framework for designing and coordinating sport scenarios for socially assistive robot based on motivational instruction patterns. We have introduced the key concepts and components that will help to guide the design of scenarios across different application domains.

470 Furthermore, we have presented three different sport scenarios where we already use our proposed framework. We hope that in the future our approach can be applied to evaluate different scenarios using different robots which are based on the same underlying interaction models. Using some standard measures (i.e. Godspeed Questionnaire and task measures) it might be possible to a) either evaluate different robots (i.e. comparing Nao and iCub) instructing on the same task using the same model, b) evaluating the same robot with different configurations of the instructional model (i.e. including reparation or acknowledgment), or c) using the same robot and the same instructional model in different domains (i.e. indoor cycling, rowing, body weight training). By implementing a structured approach of evaluating different interaction configurations, robot platforms or domains we will gain a better insight in the underlying psychological and interactional concepts that shape HRI. Thus, it might ease the task to 
implement a suited SAR to assist people on rehabilitation or everyday tasks. We have taken the first steps to systematically investigate the different aspects of interactional motivation that SAR can incorporate by showing that a simple acknowledgment by the robot can lead to higher motivation for the user which is promising for future applications of SARs.

\section{Acknowledgments}

This research/work was supported by the Cluster of Excellence Cognitive Interaction Technology 'CITEC' (EXC 277) at Bielefeld University, which is funded by the German Research Foundation (DFG).

\section{References}

[1] Icek Ajzen. The theory of planned behavior. Organizational behavior and human decision processes, 50(2):179-211, 1991.

[2] Albert Bandura. Social foundations of thought and action: A social cognitive theory. Prentice-Hall, Inc, 1986.

[3] Christoph Bartneck, Dana Kulić, Elizabeth Croft, and Susana Zoghbi. Measurement instruments for the anthropomorphism, animacy, likeability, perceived intelligence, and perceived safety of robots. International journal of social robotics, 1(1):71-81, 2009.

[4] Timothy W Bickmore and Rosalind W Picard. Establishing and maintaining long-term human-computer relationships. ACM Transactions on Computer-Human Interaction (TOCHI), 12(2):293-327, 2005.

[5] Jeanie Chan and Goldie Nejat. Social intelligence for a robot engaging people in cognitive training activities. International Journal of Advanced Robotic Systems, 9, 2012. 
[6] M. Csikszentmihalyi. Beyond Boredom and Anxiety: Experiencing Flow in Work and Play. Jossey-Bass, 25th anniversary edition, April 2000. ISBN 0787951404. URL http://www.worldcat.org/isbn/0787951404.

[7] Juan Fasola and Maja J Matarić. Socially assistive robot exercise coach: motivating older adults to engage in physical exercise. In Experimental Robotics, pages 463-479. Springer, 2013.

[8] David Feil-seifer and Maja J Matari. Defining socially assistive robotics. In in Proc. IEEE International Conference on Rehabilitation Robotics (ICORR05, pages 465-468, 2005.

[9] Deborah L Feltz, Samuel T Forlenza, Brian Winn, and Norbert L Kerr. Cyber buddy is better than no buddy: A test of the köhler motivation effect in exergames. GAMES FOR HEALTH: Research, Development, and Clinical Applications, 3(2):98-105, 2014.

[10] R. W. Scholl J. E. Barbuto. Motivation sources inventory: development and validation of new scales to measure an integrative taxonomy of motivation. In Psychological Reports, volume Vol. 82 (3), page 10111022, 1998.

[11] Chandimal Jayawardena, I-Han Kuo, Elizabeth Broadbent, and Bruce A MacDonald. Socially assistive robot healthbot: Design, implementation, and field trials. 2014.

[12] Deborah Kendzierski and Kenneth J DeCarlo. Physical activity enjoyment scale: Two validation studies. Journal of Sport $\&$ Exercise Psychology, 1991.

[13] Cory D. Kidd and Cynthia Breazeal. Robots at home: Understand530 ing long-term human-robot interaction. In 2008 IEEE/RSJ International Conference on Intelligent Robots and Systems, September 22-26, 2008, Acropolis Convention Center, Nice, France, pages 3230-3235, 2008. doi:

(1) 10.1109/IROS.2008.4651113. URL http://dx.doi.org/10.1109/IROS. 2008.4651113 
[14] Iolanda Leite, André Pereira, Ginevra Castellano, Samuel Mascarenhas, Carlos Martinho, and Ana Paiva. Modelling empathy in social robotic companions. In International Conference on User Modeling, Adaptation, and Personalization, pages 135-147. Springer, 2011.

[15] Daniel Leyzberg, Samuel Spaulding, and Brian Scassellati. Personalizing

[19] Cees Midden and Jaap Ham. Using negative and positive social feedback from a robotic agent to save energy. In Proceedings of the 4 th international conference on persuasive technology, page 12. ACM, 2009.

[20] Arne Nordmann, Sebastian Wrede, and Jochen Steil. Modeling of Movecific Languages. In International Conference on Automation and Robotics, 2015 . 
[21] E. Schegloff. When "others" initiate repair. In Applied Linguistics, volume 21(2), page 205243, 2000.

[22] S. Schneider, I. Berger, N. Riether, S. Wrede, and B. Wrede. Effects of different robot interaction strategies during cognitive tasks. In ICSR, volume 7621 of Lecture Notes in Computer Science, pages 496-505. Springer, 2012.

[23] MD Sharul. A visual formalism for complex systems. Science of Computer Programming, 8, 1987.

[24] Luise Süssenbach. Interaction and motivation in fitness communication, 2011.

[25] Luise Süssenbach, Nina Riether, Sebastian Schneider, Ingmar Berger, Franz Kummert, Ingo Lütkebohle, and Karola Pitsch. A robot as fitness companion: towards an interactive action-based motivation model. 2014.

575 [26] Johannes Wienke and Sebastian Wrede. A middleware for collaborative research in experimental robotics. In SII2011. IEEE, 2011. 